\title{
Erratum: probing top-Higgs non-standard interactions at the LHC
}

\section{Degrande, ${ }^{a, b}$ J.-M. Gérard, ${ }^{b}$ C. Grojean, ${ }^{c}$ F. Maltoni ${ }^{b}$ and G. Servant ${ }^{c, d}$}

${ }^{a}$ Department of Physics, University of Illinois at Urbana-Champaign, 1110 W. Green Street, Urbana, IL 61801, U.S.A.

${ }^{b}$ Centre for Cosmology, Particle Physics and Phenomenology (CP3), Chemin du Cyclotron 2, Université catholique de Louvain, Belgium

${ }^{c}$ CERN Physics Department, Theory Division, CH-1211 Geneva 23, Switzerland

${ }^{d}$ Institut de Physique Théorique, CEA/Saclay, F-91191 Gif-sur-Yvette Cédex, France

E-mail: cdegrand@illinois.edu, jean-marc.gerard@uclouvain.be, christophe.grojean@cern.ch, fabio.maltoni@uclouvain.be, geraldine.servant@cern.ch

ERRATUM TO: JHEP07(2012)036

The one-loop correction from $\mathcal{O}_{h g}$ to the operator $\mathcal{O}_{H G}$ is not finite, as previously stated, but diverges logarithmically. Its one-loop contribution can be written as

$$
\delta c_{H G}=\frac{g_{s} y_{t}}{4 \pi^{2}} \Re c_{h g} \log \left(\frac{\Lambda^{2}}{m_{t}^{2}}\right) .
$$

This expression should replace eq. (3.2). Consequently, eq. (3.8) should be replaced by

$$
\delta c_{H G} \approx 0.1 \Re c_{h g}-0.006 c_{y} .
$$

where we have taken $m_{t}=174.3 \mathrm{GeV}, m_{H}=125 \mathrm{GeV}, v=246 \mathrm{GeV}, \Lambda=1 \mathrm{TeV}$ and $g_{s}=1.2$ and figure 8 should be replaced by figure 1 .

\section{Acknowledgments}

We would like to thank C. Zhang for pointing out a mistake in our original computation of the one-loop corrections to $\mathcal{O}_{H G}$. 

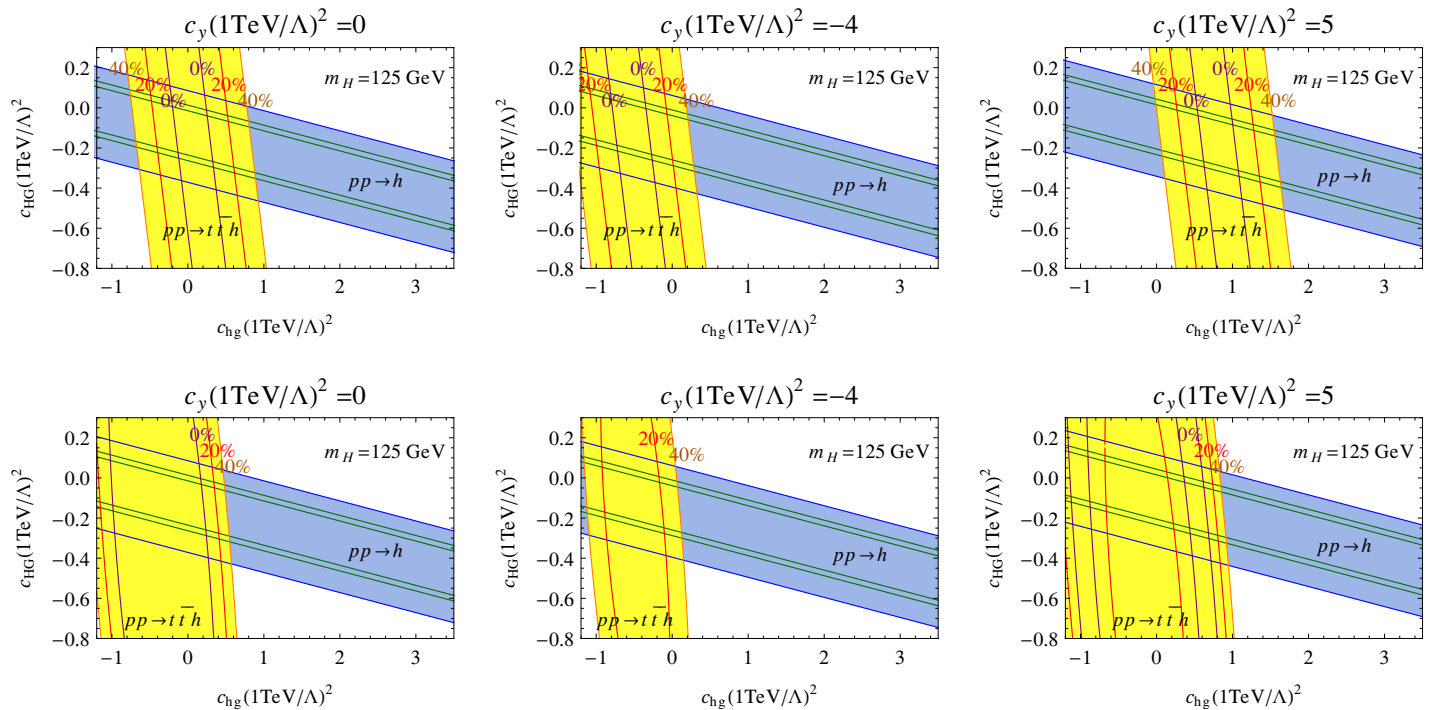

Figure 1. In blue, the region allowed by the Higgs production constraints at $7 \mathrm{TeV}$ for $m_{H}=$ $125 \mathrm{GeV}$. The green lines delimit the 2 allowed tiny bands obtained if the Higgs cross-section is measured at its SM value with a precision of $20 \%$. The yellow region is obtained by assuming a $40 \%$ precision on the $t \bar{t} h$ cross-section at $14 \mathrm{TeV}$ with the measured central value matching the SM prediction and $c_{G}=0$. The three plots correspond respectively to $c_{y}(\mathrm{TeV} / \Lambda)^{2}=0,-4,+5$. The upper plots are obtained when neglecting the $\mathcal{O}\left(1 / \Lambda^{4}\right)$ terms in the $t \bar{t} H$ cross section. The bottom plots instead include these higher order terms. 